\title{
Dissipative parametric modulation instability and pattern formation in nonlinear optical systems
}

 \\ ${ }^{a}$ Aston Institute of Photonic Technologies, Aston University, Birmingham B4 7ET, UK. \\ ${ }^{\mathrm{b}}$ Departament de Fisica i Enginyeria Nuclear, Universitat Politècnica de Catalunya, E-08222, \\ Barcelona, Spain. \\ 'Institute of Computational Technologies SB RAS, Novosibirsk, 630090, Russia. \\ ${ }^{\mathrm{d}}$ Novosibirsk State University, Novosibirsk, 630090, Russia. \\ eInstitució Catalana de Recerca i Estudis Avançats, Passeig Lluis Companys 23, E-08010, \\ Barcelona, Spain.
}

\begin{abstract}
We present the essential features of the dissipative parametric instability, in the universal complex GinzburgLandau equation. Dissipative parametric instability is excited through a parametric modulation of frequency dependent losses in a zig-zag fashion in the spectral domain. Such damping is introduced respectively for spectral components in the $+\Delta F$ and in the $-\Delta F$ region in alternating fashion, where $F$ can represent wavenumber or temporal frequency depending on the applications. Such a spectral modulation can destabilize the homogeneous stationary solution of the system leading to growth of spectral sidebands and to the consequent pattern formation: both stable and unstable patterns in one- and in two-dimensional systems can be excited. The dissipative parametric instability provides an useful and interesting tool for the control of pattern formation in nonlinear optical systems with potentially interesting applications in technological applications, like the design of modelocked lasers emitting pulse trains with tunable repetition rate; but it could also find realizations in nanophotonics circuits or in dissipative polaritonic Bose-Einstein condensates.
\end{abstract}

Keywords: Nonlinear instabilities, pattern formation, mode-locking

\section{INTRODUCTION}

Instabilities of the stationary states of nonlinear systems are the fundamental and essential ingredients from which qualitatively different states of the systems can be achieved. The linear stage of the instabilities are associated with the exponential growth of some spatial or temporal modes. When the growing sidebands have reached a sufficiently big magnitude the original qualitative features of system the can be modified substantially and the system can evolve into a new and qualitatively different stable state or even do not reach a stationary state resulting in a less predictable and more chaotic dynamics. Spatial, temporal or spatiotemporal patterns can emerge in the nonlinear stages of instabilities establishing a new order and new correlations between different parts of the system. ${ }^{1}$

Pattern forming instabilities can take place in a great variety of different physical, chemical and biological systems ${ }^{2}$ such as fluids, plasmas, chemical reactions, bacteria populations, Bose-Einstein condensates and lasers where we can have both spatial patterns for instance in the transverse plane of the resonator ${ }^{3}$ or longitudinal patterns, as for instance, pulse trains.

On the contrary, in engineering, instabilities are seen more often as a detrimental factor which could limit or damage the performances of a system and make it less functional and appealing. Hence an effort is done in order to suppress instabilities and/or to control them since they could disturb the regular operation of technological devices. ${ }^{4-6}$ Nevertheless, in the last years, there has been an increasingly growing trend especially in the fiber optics community which aims to exploit instabilities as a source of new interesting and useful behaviors. In

Further author information: (Send correspondence to A. M. Perego), E-mail: peregoa@aston.ac.uk, Telephone: +44 1212043512 .

Nonlinear Optics and its Applications IV, edited by Benjamin J. Eggleton, Neil G. R. Broderick,

Alexander L. Gaeta, Proceedings of SPIE Vol. 9894, 98940A · ¿ 2016 SPIE

CCC code: $0277-786 X / 16 / \$ 18 \cdot$ doi: $10.1117 / 12.2225595$

Proc. of SPIE Vol. 989498940 A-1 
particular it has been shown that the Faraday (also called parametric) instability due to the periodic, along the spatial propagation coordinate, modulation of group velocity dispersion and/or nonlinearity coefficient is able to destabilize the temporally homogeneous field state and to initiate the exponential growth of spectral sidebands. ${ }^{7,8}$

Note that while in the original Faraday experiment ${ }^{9}$ or in other realizations in spatially extended systems, ${ }^{10}$ the modulation of a parameter in time induces the formation of a pattern in space, viceversa in the case of optical fibers the modulation of a parameter in space excites a pattern in time.

Theoretical and numerical studies of the Faraday instability excited by modulation of dispersion and of nonlinearity in ring fiber resonators have demonstrated that the generation of a stable and regular temporal pattern (pulse train) is possible. ${ }^{11,12}$ Nevertheless no experimental confirmation of the generation of pulse trains thanks to this technique has been reported so far, probably due to the fact in the nonlinear stage of the instability a mechanism to suppress the interpulse noise is missing.

The increasing demand of pulsed laser sources with tunable properties (width, intensity peak, repetition rate) motivates a further effort in designing new versatile solutions.

In this paper we describe in detail a recently proposed scheme of dissipative parametric modulation, achieved through spectrally dependent zig-zag filtering, which can be implemented both in nonlinear fiber lasers leading to the generation of stable pulse trains, and in spatially extended optical systems too, giving rise in that case to pattern formation in one and in two spatial dimensions. ${ }^{13}$ The generated coherent structures undergo a self-similar periodic evolution that will be characterized in the following sections and are the new self-organized stable state achieved in the nonlinear stage of the instability.

\section{DISSIPATIVE PARAMETRIC MODULATION INSTABILITY: THE LINEAR STAGE}

We describe now precisely how the dissipative parametric modulation instability can be excited and which are its distinguishing features. We consider the universal model, ubiquitous in many fields of science, the complex Ginzburg-Landau equation (CGLE) ${ }^{14}$ as the master equation describing the dynamics of a generic nonlinear spatially extended system. For a one-dimensional system the CGLE reads:

$$
\frac{\partial A}{\partial t}=\mu A+(b-i d) \frac{\partial^{2} A}{\partial x^{2}}+(i c-s)|A|^{2} A .
$$

Where the complex field envelope $A(x, t)$ is defined in space $x$ and evolving in time $t$, and the coefficients $\mu, c, b$, $d$ and $s$ describe respectively gain, nonlinearity, diffusion diffraction and gain saturation. We consider the case of Benjamin-Feir stable CGLE and to guarantee this fact we choose $c, d>0$. In this case the field homogeneous solution is given by $A_{h s}=A_{0} \exp \left(i c\left|A_{0}\right|^{2}\right)$ where $\left|A_{0}\right|^{2}=\mu / s$.

The dissipation modulation scheme is the following: we consider first a nonlinear evolution for a temporal interval $T_{f} / 2$ described by Eq.1 then we apply losses in the wavenumber region $+\Delta k$ by multiplying the field with a spectral filter whose transmittance peak is located in the symmetric wavenumber region $-\Delta k$ then a new nonlinear evolution described by Eq.1 takes place followed by spectral losses applied in the wavenumber region $-\Delta k$ this time multiplying the field with a spectral filter whose transmittance peak is located in the wavenumber region $+\Delta k$. Such a sequence defines the modulation period $T_{f}$. This surprisingly simple modulation scheme causes, after many modulation periods, an exponential average growth of modulation modes with wavenumbers $\pm k_{m}$.

If we monitor the temporal evolution of the maximally growing modes $\pm k_{m}$ we observe that they both grow in average but oscillating in antiphase: this is due to the fact that periodically each one of the two modes is abruptly damped by the action of the spectral filter, but the spectral filters act separately on the two modes so that when one is damped the other is basically unaffected. After one mode has been damped, the Four-Wave-Mixing process due to the coupling between the "surviving" mode and the homogeneous $(k=0)$ mode, provides energy transfer for the growth of the recently damped one. This evolution can be clearly observed in Fig. 1. Such a dynamics is different with respect to the growth process of sidebands modes in both well-known Benjamin-Feir and classical dispersive Faraday instability: in particular, in the former the growth is monotonic, while in the latter it is oscillatory, but the most unstable modes $\pm k_{m}$ oscillate in phase. ${ }^{13}$ We are hence in presence of a new growth dynamics which makes the dissipative parametric instability through zig-zag modulation of the dissipation for different spectral components a unique example in the landscape of nonlinear instabilities. 
A further insight into the nature of the dissipative parametric instability can be achieved by studying how the maximally growing modes depend on the parameters of the system, in particular on the modulation period $T_{f}$ and on the field intensity $|A|^{2}$. An estimation of the instability frequency can be achieved by imposing a parametric resonance condition between the modulation frequency and the spectrum of the Bogoliubov excitations of the CGLE. From the parametric resonance condition we can expect that the value of $k_{m}$, actually the first excited mode, can be obtained form the nonlinear dispersion relation $k\left(\omega_{f} / 2\right)$ where $\omega_{f}=2 \pi / T_{f}$. Under suitable approximations, in the long-wavelength limit one can obtain the formula for the maximally unstable mode scaling:

$$
k_{m} \approx \frac{\pi}{T_{f} \sqrt{2 c d\left|A_{0}\right|^{2}}}
$$

We have compared the analytical predictions with the results of the Floquet linear stability analysis of the homogeneous state with respect to small modulation modes. The predictions of the Floquet analysis are valid until the amplitude of the modulation modes are much smaller than the homogeneous one. Since we are in presence of a periodic system an analysis of the stability of each spectral mode $k$ can be performed by computing numerically the evolution of small complex perturbations to the complex sidebands amplitudes $a_{+}(k)$ and $a_{-}(-k)$ for one modulation period. Considering $\operatorname{Re}\left(a_{+}(k)\right), \operatorname{Im}\left(a_{+}(k)\right), \operatorname{Re}\left(a_{-}(-k)\right)$ and $\operatorname{Im}\left(a_{-}(-k)\right)$ after an one modulation period evolution, we can build a stability matrix for the mode $k$ which provides information on the perturbations growth or damping. In particular the matrix eigenvalues for the mode $k$ are called Floquet multipliers for that mode and if at least one of theirs absolute values is greater than 1 then a modulation of the homogeneous solution with wavenumber $k$ will grow exponentially. Repeating the procedure for all $k \mathrm{~s}$ we can obtain the instability spectrum for a given set of parameters. An instability map as a function of a system parameter can be computed accordingly. We show here an example of an instability map obtained varying the modulation period $T_{f}$ (see Fig.1). The analytical prediction is plotted with the dashed black line evaluating Eq.2 with the field intensity averaged over one modulation period (indeed in presence of strongly detuned spectral filters the homogenous mode can be slightly damped giving and the analytical homogeneous solution overestimates the effective average intensity).

a)

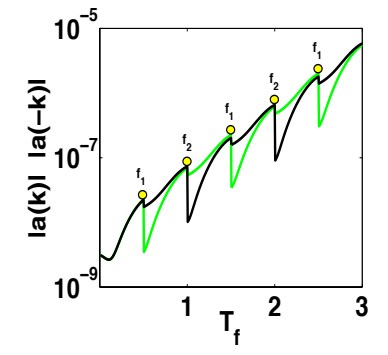

b)



Figure 1. The average growth process of the symmetric sidebands amplitudes absolute values, black and green lines, is depicted in a) showing the alternating big damping due to the action of the filters (denoted with yellow circles and the symbols $f_{1}$ and $f_{2}$ ) and successive growth. In b) we have an example of an instability map, where the maximum absolute value of the Floquet multipliers is plotted in $\left(k, T_{f}\right)$ space; instability takes place in the colored regions while the dashed black line describes the analytic prediction for the maximally growing mode. 
We have used without loss of generality Supergaussian detuned spectral filters of order 4 in the one dimensional study while simple Gaussian filters in the case of the two-dimensional patterns discussed in Section 3 . In general a minimum spectral detuning between the filters must be present in order to achieve the instability while an excessive detuning is detrimental also because it damps completely the homogeneous mode. In the regime where the instability is present an increase of the filters width or a reduction of their detuning excites smaller wavenumbers. More details on the filters, parameters used and features of the dissipative parametric instability by zig-zag modulation of the dissipation for different spectral components can be found in Ref.13.

\section{DISSIPATIVE PARAMETRIC MODULATION INSTABILITY: THE NONLINEAR STAGE AND PATTERN FORMATION}

In the nonlinear stage of its evolution, the dissipative parametric instability can lead to pattern formation. In general both regular and stable but also irregular and dynamically varying patterns can be generated. In the latter case, processes of creation and annihilation of coherent structures occur repeatedly. The spectral filters play also an important role in the nonlinear stage by suppressing the noise and keeping the pattern stable. Hence the zig-zag spectral filtering acts an effective distributed saturable absorber. A detailed study on the sufficient and necessary conditions for achieving a stable pattern formation has not been obtained yet and it is left for future studies but we have noticed that even though the instability is present with different filters shapes, it looks like that steeper decay of the filter spectral profiles can help in achieving more stable patterns.

Examples of one- and two-dimensional patterns are provided in Fig.2. Stable patterns undergo and internal periodic dynamics: during the nonlinear evolution in presence of gain the coherent structures evolve into parabolic shape like similaritons in optical amplifiers, later the action for the spectral filter narrows them bringing them closer to a Gaussian shape. This phenomenon occurs both in the case of one- and two-dimensional patterns. ${ }^{13}$

a)

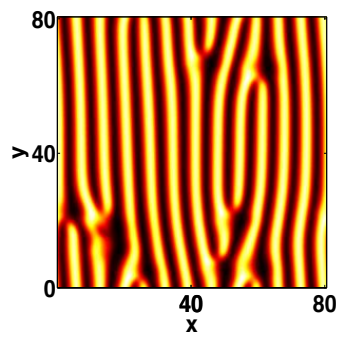

b)

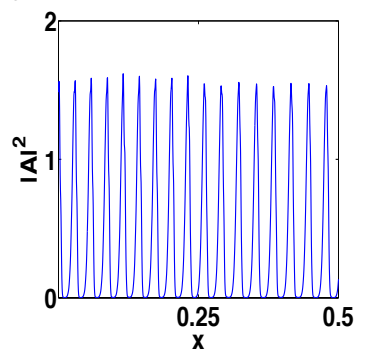

Figure 2. Example of two- and one-dimensional patterns in a) and b) respectively; axis are in arbitrary units. Both pictures are taken in the stationary state just after the action of one filter.

\section{APPLICATIONS}

The dissipative parametric instability has been discussed up to now in the very general and universal mathematical framework provided by the CGLE. We comment now some of the possible physical and technological implementations where the dissipative parametric instability could be observed and lead to useful performances of the systems. In the first case it is worth to mention its great potentiality as a tool to create mode locked lasers. Especially concentrating on fiber lasers or amplifiers we have that for those devices a mathematical description of the dynamics is provided by a generalized nonlinear Schroedinger equation (GNLSE) whose salient features 
in terms of relevant physical effects are the ones of the CGLE (Eq.1) but with temporal and spatial coordinates interchanged, we have in particular:

$$
\frac{\partial A}{\partial z}=\frac{g}{2} A+\left(B-i \frac{\beta_{2}}{2}\right) \frac{\partial^{2} A}{\partial t^{2}}+(i \gamma-\delta)|A|^{2} A
$$

where $\beta_{2}$ is the group velocity dispersion, $\gamma$ the nonlinearity coefficient, $g$ is the gain coefficient and $\delta$ its saturation parameter, while $B$ describes the finite gain bandwidth. To observe the dissipative parametric instability in fiber optics we can just consider a zig-zag spectral filtering periodic in space and based on filters (fiber Bragg gratings for instance) whose reflectivity profile in $\omega$ (temporal frequency) domain is detuned, in this case a simple linear cavity laser can be realized (See Fig.3). The pulse repetition rate could be tuned by varying the filters detuning, the pump power or the cavity length.

Due to the passive nature of the dissipation modulation, which does not require an external drive, this mechanism could be potentially implemented to achieve generation of pulses in nanophotonic circuits.

Other systems where the dissipative parametric instability could be observed are for instance self-imaging resonators where wavenumbers located in spectral regions $\pm \Delta k$ are filtered in alternating fashion, or by alternating gain and filtering sections in wavenumber domain as depicted schematically in Fig.3.


Figure 3. Two schematic representations of possible implementations of the zig-zag spectral filtering in wavenumber domain a) and in temporal frequency $\omega$ domain b) where $L$ and $R$ stand respectively for dissipated and reflected components. The scheme presented in b) applies well to linear cavity fiber lasers with frequency detuned FBGs reflectivity profile so that the back and forth propagating light will experience the proper dissipation modulation.

Concerning further implementations one can think about dissipative Bose-Einstein condensates, in particular exciton-polariton condensates in semiconductor microcavities. The dissipative nature of polaritonic condensates makes them good candidates for the observation of the dissipative parametric instability, in this case the dynamics is described by an equation of the CGLE type too ${ }^{15}$ and the wavenumber dependent losses could be implemented simply by using cavity Bragg mirrors with detuned reflectivity profiles in wavenumber space.

\section{CONCLUSIONS}

In conclusion we have presented the main features of the recently proposed dissipative parametric instability due to zig-zag filtering for different spectral components. The key features of the instability in the linear stage have been described and compared with those of other known instabilities. Examples of the pattern formation induced by the dissipative parametric instability in one and two-dimensional systems have been given too. Finally potential implementations of the instability and related generation of coherent structures have been discussed with particular emphasis to nonlinear fiber optics where there is an increasing demand for new tools for the generation of pulses, but also including very different systems: this shows the potentiality of the dissipative parametric instability to have universal applications that can even range outside the field of nonlinear optics. 


\section{ACKNOWLEDGMENTS}

We acknowledge support from the Spanish Ministerio de Educación y Ciencia, the European FEDER Project No. FIS2011-29731-C02-01, the ERC project ULTRALASER, the Russian Ministry of Education and Science (Grant No. 14.B25.31.0003), the Russian Foundation for Basic Research (Grant No. 15-02-07925), a Presidential Grant for Young Researchers (Grant No. 14.120.14.228-MK), the Dinasty Foundation and the NATO SPS (Grant No. 985048). N.T. is supported by the Russian Science Foundation (Grant No. 14-21-00110). A. M. P. acknowledges support from the ICONE Project through the Marie Curie Grant No. 608099.

\section{REFERENCES}

[1] Cross, M. C. and Hohenberg, P. C., "Pattern formation outside of equilibrium," Rev. Mod. Phys. 65, 851 (1993).

[2] Tlidi, M., Staliunas, K., Panajotov, K., Vladimirov, A. G., and Clerc, M. G., "Localized structures in dissipative media: from optics to plant ecology," Phil. Trans. R. Soc. A 372, 2027 (2014).

[3] Staliunas, K. and Sánchez-Morcillo, V. J., [Transverse patterns in nonlinear optical resonators], Springer, Berlin (2003).

[4] Kumar, S., Herrero, R., Botey, M., and Staliunas, K., "Taming of modulation instability by spatio-temporal modulation of the potential," Scientific Reports 5, 13268 (2015).

[5] Ahmed, W. W., Kumar, S., Herrero, R., Botey, M., Radziunas, M., and Staliunas, K., "Stabilization of flatmirror vertical-external-cavity surface-emitting lasers by spatiotemporal modulation of the pump profile," Phys. Rev. A 92, 043829 (2015).

[6] Kumar, S., Herrero, R., Botey, M., and Staliunas, K., "Suppression of modulation instability in broad area semiconductor amplifiers," Opt. Lett. 39, 5598-5601 (2014).

[7] Smith, N. and Doran, N. J., "Modulational instabilities in fibers with periodic dispersion management," Opt. Lett. 21, 570-572 (1996).

[8] Abdullaev, F. K., Darmanyan, S. A., Bischoff, S., and Sørensen, M. P., "Modulational instability of electromagnetic waves in media with varying nonlinearity," J. Opt. Soc. Am. B 14, 27-33 (1997).

[9] Faraday, M., "On a peculiar class of acoustical figures; and on certain forms assumed by a group of particles upon vibrating elastic surfaces," Phil. Trans. R. Soc. London 121, 299-318 (1831).

[10] Staliunas, K., Longhi, S., and de Valcárcel, G. J., "Faraday patterns in bose-einstein condensates," Phys. Rev. Lett. 89, 210406 (2002).

[11] Staliunas, K., Hang, C., and Konotop, V. V., "Parametric patterns in optical fiber ring nonlinear resonators," Phys. Rev. A 88, 023846 (2013).

[12] Conforti, M., Mussot, A., Kudlinski, A., and Trillo, S., "Modulational instability in dispersion oscillating fiber ring cavities," Opt. Lett. 39, 4200-4203 (2014).

[13] Perego, A. M., Tarasov, N., Churkin, D. V., Turitsyn, S. K., and Staliunas, K., "Pattern generation by dissipative parametric instability," Phys. Rev. Lett. 116, 028701 (2016).

[14] Aranson, I. and Kramer, L., "The world of the complex ginzburg-landau equation," Rev. Mod. Phys. 74, 99 (2002).

[15] Carusotto, I. and Ciuti, C., "Quantum fluids of light," Rev. Mod. Phys. 85, 299 (2013). 